\title{
Effects of a new nutraceutical combination on cognitive function in hypertensive patients
}

\author{
Giuseppe Giugliano ${ }^{1}$, Alessia Salemme ${ }^{2}$, Sara De Longis ${ }^{3}$, Marialuisa Perrotta ${ }^{4}$, Valentina D'Angelosante ${ }^{4}$, \\ Alessandro Landolfi ${ }^{4}$, Raffaele Izzo ${ }^{3 *}$ (i) and Valentina Trimarco ${ }^{5}$
}

\begin{abstract}
Background: Chronic increased arterial blood pressure has been associated with executive dysfunction, slowing of attention and mental processing speed, and later with memory deficits. Due to the absence of a concrete therapeutic approach to this pathophysiological process, in the last decades there has been an increasing interest in the use of nutraceuticals, especially those with antioxidant properties, which own strong neuroprotective potential, that may help to improve cognitive function and to delay the onset of dementia.

Results: We evaluated the effects of the treatment with a new nutraceutical preparation containing different molecules with potent antioxidant properties (AkP05, IzzeK ${ }^{\circledast}$ ) and placebo on a cohort of thirty-six hypertensive patients. At baseline, neuropsychological evaluation, arterial stiffness and biochemical parameters of the subjects were comparable. After 6 months of treatment, there was a significant reduction of the augmentation index in the AkP05-treated group. Moreover, the measurement of cognitive function, evaluated with MoCA test and Word Match Testing, showed a significant improvement in patients receiving the active treatment. In addition, the group treated with nutraceutical reached a better Stroop test score, while subjects that received placebo did not showed any improvement. Finally, a positive relationship between SBP variation and the psychometric assessment with the EQ-VAS scale was observed only in the active treatment group.

Conclusions: In this study, we demonstrated that the therapy with a new nutraceutical preparation is able to significantly increase the scores of important neuropsychological tests in hypertensive patients already on satisfactory blood pressure control. Although future studies are needed to better characterize the molecular mechanisms involved, these results candidate the new nutraceutical combination as a possible therapeutic strategy to support the cerebrovascular functions and delay the onset of dementia in hypertensive patients.
\end{abstract}

Keywords: Nutraceutical, Neuropsychological evaluation, Arterial stiffness

\section{Background}

One of the most common neurological disorders in the elderly is represented by a progressive and unremitting deterioration of cognitive functions, a condition globally referred to as dementia $[1,2]$. In consideration of the lack of effective treatments and the demographic conditions shifting the population toward older adults, the incidence of dementia is predicted to exponentially increase in the

\footnotetext{
* Correspondence: raffaele.izzo@unina.it

${ }^{3}$ Hypertension Research Center; Department of Translational Medical

Sciences, Federico II University, via Pansini 5, 80131 Naples, Italy

Full list of author information is available at the end of the article
}

future. Basically, for the vast majority of cases, cognitive impairment can be ascribed to two main causes: Alzheimer's Disease $(\mathrm{AD})$ and cerebrovascular diseases. Although it has been generally thought that $\mathrm{AD}$ represents those cases of dementia related to genetic alterations that leads to an increased production of the $A \beta$ (amyloid $\beta$ peptide) and accumulation of plaques, we are now aware that a clear-cut distinction of $\mathrm{AD}$ and cerebrovascular diseases is not always found in the clinical practice, having patients a mixture of both pathologies [3]. On this notice, experimental models have shown that, even when no evident increase of $A \beta$ production does exist, alterations of 
cerebrovascular homeostasis can determine a failure in the mechanisms responsible for the clearance of $A \beta$ from the brain [4-6]. Thus, the definition of vascular cognitive impairment (VCI) predicts to recapitulate a wide range of cognitive deficits caused by vascular factors, and contributing to the development of later dementia, a condition where cognitive decline is irreversible and impairs even day-to-day functioning [7]. It is generally accepted that, whichever the primary cause, the outcome toward dementia and/or $\mathrm{AD}$ is a stage where no strategy is available to improve or at least counteracts the symptomatic manifestations of the disease.

Hypertension, a highly prevalent disease, representing nowadays one of the most impacting cause of disease burden and disability, is the major risk factor for cerebrovascular diseases [1]. Chronic increased arterial blood pressure has been associated with executive dysfunction, slowing of attention and mental processing speed, and later with memory deficits. Similarly, experimental models of hypertension allowed to unravel several pathophysiological mechanisms involved in this deleterious association $[4,8-10]$. In addition, the intertwining of cerebrovascular diseases and $\mathrm{AD}$ can be also inferred from the neuropathological hallmarks of the two conditions, showing amyloid plaques and neurofibrillary tangles but also microvascular and ischemic lesions.

Taken together the neuropathological traits of brains affected by dementia have suggested over years the existence of an oxidative and inflammatory burden, deriving from the molecular lesions identified. Recent evidence suggests that immune and inflammatory processes significantly contribute to the progression of pathological hallmarks [11-15]. On a similar notice, inflammation and immune activation are clearly recognized as crucial aspects of hypertension and related target organ damage $[16,17]$.

The idea to counteract the inflammatory burden in early stages of cognitive deterioration and in those predisposing conditions, like hypertension and vascular risk factors, where pro-inflammatory and oxidative mechanisms have a considerable impact as well, raised an expanding area of research aimed at testing the potential beneficial effects of therapeutic strategies enhancing the global anti-oxidant and anti-inflammatory power. On this notice, several evidence in animal models support the use of combined nutraceutical supplementations in the prevention of cognitive decline [18-21]. At this regard different molecules with potent antioxidant properties, including Gingko biloba, extract of Bacopa monniera, Phosphatidylserine, a phospholipid component of the neuronal membrane, Green tea polyphenols and Epigallocathechin, although with mixed results, have shown to have positive effects on cognitive function also in humans [22-36].

According to these promising results, the present study was planned to asses in a double blind, parallel group versus placebo, the effects of a chronic treatment with a novel nutraceutical combination containing dry extract of Gingko biloba, Bacopa monniera, Green tea, phosphatidylserine and catechins on blood pressure, metabolic profile, arterial stiffness and cognitive function in hypertensive patients with a satisfactory blood pressure control obtained by an adequate antihypertensive therapy.

\section{Methods \\ Study design}

The study was conducted in accordance with the guidelines of the declaration of Helsinki and was approved by the Ethic Committee of the IRCCS Neuromed, Pozzilli (Isernia), Italy, which designed and registered the study protocol (ClinicalTrials.gov ID: NCT02572219). Written informed consent was obtained from each subject.

This was a randomized, double blind, placebo-controlled study with a 6-months follow-up, during which patients were randomized to receive placebo or active treatment of a nutraceutical preparation (AkP05, IzzeK ${ }^{\circ}$ ) containing: dry plant extract of Bacopa monnieri $300 \mathrm{mg}+$ dry extract of Ginkgo biloba leaves $50 \mathrm{mg}+$ Phosphatidylserine $25 \mathrm{mg}+$ dry extract of green tea leaves $40 \mathrm{mg}$ + Catechin $20 \mathrm{mg}$. Akademy Pharma produced the nutraceutical preparation. The company also produced the placebo, similar in appearance and organoleptic properties to the nutraceutical preparation.

Thirty-six patients were enrolled in the study between January 2017 and March 2017. Subjects of both sexes aged between 40 and 70 with diagnosis of essential hypertension were screened for assessment of the following exclusion criteria. Exclusion criteria were any of the following: previous cardiac or cerebrovascular event; heart failure; diabetes mellitus; history of atrial fibrillation or other severe arrhythmias; chronic kidney disease (defined as serum creatinine levels $>1.4 \mathrm{mg} / \mathrm{dL}$ ); preexisting psychiatric disorders; neurodegenerative diseases such as multiple sclerosis, amyotrophic lateral sclerosis, Parkinson's disease, early onset/genetic Alzheimer's disease, neuromuscular pathologies, epilepsy; diagnosis of dementia. In addition, patients requiring any pharmacological treatment beyond antihypertensive drugs or with intolerance to nutraceutical compounds, pregnant women and women planning to conceive were also excluded from the study. Only hypertensive patients on pharmacological therapy and with a satisfactory and stable blood pressure control were enrolled, in order to rule out the possibility of an interference of potential additional antihypertensive drugs on cognitive function.

Eighteen were assigned to the nutraceutical treatment group and 18 to the placebo group, according to a computer based randomization double-blinded scheme. 


\section{Procedures}

Clinical history, risk factors and current pharmacological therapies were assessed at the baseline evaluation. Smokers included current and former smokers. Hypertension was diagnosed if systolic arterial pressure exceeded $140 \mathrm{mmHg}$ and/or diastolic arterial pressure exceeded $90 \mathrm{mmHg}$, or if the patient was in antihypertensive drugs. Systolic and diastolic BP were measured by standard sphygmomanometer after $5 \mathrm{~min}$ in the supine position, according to the guidelines of the European Society of Hypertension/ European Society of Cardiology [37].

All patients suitable for enrollment underwent 2 prerandomization visits: at baseline and after 2 weeks of run-in period in order to assess the stable and satisfactory blood pressure control. At the end of the run-in period, all randomized patients underwent the following evaluations:

- Neuropsychological evaluation: a battery of neuropsychological tests was administered to profile specific aspects of cognitive domains such as associative memory, visual-spatial memory, working memory, attentive skills, and reasoning skills. Cognitive assessment was administered by a welltrained psychologist (A.S.). The specific tests used were: the Montreal Cognitive Assessment (MoCA); Word pairing learning test; Stroop test; Visual analogue scale (EQ-VAS). The execution of all the neuropsychological tests was completed always in the same order.

- Evaluation of arterial stiffness was obtained by measuring the augmentation index (AI) with the SphygmoCor pulse wave analysis system (AtCor Medical Pty. Ltd., Sydney, Australia).

- Peripheral blood sampling for assessment of glucose and lipid profile.

\section{Neuropsychological tests Montreal cognitive assessment (MoCA)}

This test was designed as a tool for screening of mild cognitive deterioration and has been validated as a gold standard evaluation in vascular-related dementias [38]. The specific cognitive subdomains assessed are the following: attention, executive functions, memory, language, visuospatial abilities. The MoCA administration time is $10 \mathrm{~min}$. The maximum possible score is 30 points; a score equal to or greater than 26 is considered normal.

\section{Word match testing}

The standard procedure requires reading a list of 10 pairs of words in the fixed order at the rate of a pair every $2 \mathrm{~s}$. Next, in a different order from the first reading, the first member of each pair is read, and the subject is asked to recall the second. This procedure is repeated for 3 times. 5 pairs of words' associations are usually easier (i.e. back and forth), while the other 5 are difficult (i.e. explosion-stamp). The test evaluates the associative memory in simple voluntary learning conditions.

\section{Stroop test}

This is a good example of the interference effect on highly automated tasks such as reading. The subject is asked to read the words in the first test, to name the colors in the second and third tests. It is necessary to mark both the mistakes made by the subject, but also the time spent in each trial. Results of the test are provided as two scores: 1) number of errors (Stroop E); and 2) number corrected for time and referred to as interference time (Stroop T).

\section{Visual analogue scale (EQ-VAS)}

Visual analogue scale is the second part of the EQ-5D test, in which the subject is asked to mark health status on the day of the interview on a $20 \mathrm{~cm}$ vertical scale with end points of 0 and 100 . There are notes at the both ends of the scale that the bottom rate (0) corresponds to "the worst health you can imagine", and the highest rate (100) corresponds to "the best health you can imagine".

Participants were allowed to take a 5 min break if necessary to minimize tiredness and maintain motivation. The average duration of the whole neuropsychological evaluation was about of $30 \mathrm{~min}$. For the first 3 tests, the scores obtained were corrected for age and level of education, following correction matrices developed according to validation rules for the Italian population [39]. Adjusted scores were then converted to a five-point interval scale, from 0 to 4 equivalent scores (ES) [39]. The five-point interval scale was divided as follows: $0=$ below average scores; 1 = low average scores; 2 = average scores; $3=$ high average scores; 4 = above average scores [39].

\section{Arterial stiffness}

AI, a validated index of arterial stiffness, was measured by applanation tonometry. To record the central pressure waveform, the indirect method of arterial tonometry was used. Briefly, pressure waveform was recorded at the left radial artery and, using the generalized transfer function, it was converted into a calculated central pressure waveform [40]. All measurements were performed with the SphygmoCor device and designated software (AtCor Medical Pty. Ltd., Sydney, Australia). SphygmoCor uses a high fidelity Millar strain-gauge transducer (Millar Instruments, Houston, TX) allowing for measurement of the first systolic peak (P1), the second systolic peak (P2), and the central pulse pressure (PP) from the calculated aortic waveform. AIx was then calculated as: $\mathrm{AI}(\%)=(\mathrm{P} 2-\mathrm{P} 1) \mathrm{PP} * 100$. All patients were maintained without smoking, alcohol or caffeine, starting from the 
night before the evaluation. All measurements were performed with the patient in supine position in a quiet temperature-controlled laboratory $\left(26 \pm 1{ }^{\circ} \mathrm{C}\right)$.

\section{Endpoints}

The primary endpoint was the effect of the two treatments (placebo vs active compound) on the score obtained at the Montreal Cognitive Assessment (MoCA) test. Secondary endpoints were the effects on other neuropsychological tests, arterial stiffness, as evaluated by SphygmoCor technology, and metabolic parameters.

\section{Statistical analysis}

Based on preliminary data and previous studies assessing the impact of hypertension on cognitive functions, we hypothesized that $50 \%$ of patients treated with the nutraceutical preparation would experience an increase of at least one equivalent score (ES) of MoCA test (primary endpoint) with respect to $10 \%$ of subjects assuming placebo.

On this assumption, a sample size of 17 subjects in each group would have provided a power of $80 \%$ with a two-tailed $\alpha$-error of $5 \%$. In addition, expecting a dropout rate of about $5 \%$, due to the documented good tolerability of nutraceutical therapies, the total number of patients enrolled was of 36 (18 in each group). Data were analyzed using SPSS (version 22.0; SPSS, Chicago, Illinois, USA) and expressed as number and percentages or means \pm SD. Differences between the two groups at baseline were evaluated by independent sample t-test or chi-square test, as appropriate. A general linear model (GLM) for repeated measures with correction for treatment was performed to evaluate the treatment effect in both groups. Pearson correlation coefficients were used to assess correlations between changes in clinical variables and changes in neuropsychological tests. For all tests, a $p$ value $<0.05$ was considered statistically significant.

\section{Results}

All the 36 patients enrolled completed the entire protocol of the study. Patients included in the study were predominantly males $(75 \%)$ with a mean age of $58.1 \pm 7.2$ years, mean body mass index of $26.9 \pm 3.0$, mean SBP of $134.4 \pm 15.1$, and mean DBP of $85.0 \pm 10.7 \mathrm{mmHg}$. At baseline, the patients of the two arms were comparable for all clinical and metabolic characteristics, arterial stiffness, and neuropsychological evaluation (Table 1). Also antihypertensive treatments were not different between the two study arms (Table 1).

After 6 months, patients were subjected to the assessment of the same clinical and biochemical variables, as well as the cognitive profile. All the variables resulted unchanged in both placebo and active treatment groups, with respect to the baseline condition (Table 2), with the exception of AI which was significantly reduced in
Table 1 Baseline characteristics according to the treatment protocol group

\begin{tabular}{|c|c|c|c|}
\hline & $\begin{array}{l}\text { Placebo } \\
(n=18)\end{array}$ & $\begin{array}{l}\text { Active treatment } \\
(n=18)\end{array}$ & $p$ \\
\hline Age (yrs) & $57.9 \pm 6.7$ & $58.2 \pm 8.0$ & 0.910 \\
\hline Males & $14(77.8)$ & $13(72.2)$ & 0.700 \\
\hline Height (cm) & $170.0 \pm 9.2$ & $170.2 \pm 8.6$ & 0.955 \\
\hline Weight (kg) & $75.9 \pm 11.0$ & $80.2 \pm 15.7$ & 0.351 \\
\hline Body Mass Index $\left(\mathrm{kg} / \mathrm{m}^{2}\right)$ & $26.3 \pm 3.1$ & $27.7 \pm 2.8$ & 0.161 \\
\hline $\mathrm{SBP}(\mathrm{mmHg})$ & $133.3 \pm 16.9$ & $135.6 \pm 13.5$ & 0.666 \\
\hline $\mathrm{DBP}(\mathrm{mmHg})$ & $84.1 \pm 12.3$ & $85.8 \pm 9.1$ & 0.636 \\
\hline Heart rate (bpm) & $68.8 \pm 6.8$ & $66.4 \pm 7.9$ & 0.350 \\
\hline Total cholesterol (mg/dl) & $198.2 \pm 23.7$ & $201.9 \pm 27.5$ & 0.667 \\
\hline HDL cholesterol (mg/dl) & $47.6 \pm 7.9$ & $51.3 \pm 7.2$ & 0.144 \\
\hline LDL cholesterol (mg/dl) & $129.2 \pm 26.1$ & $126.7 \pm 30.4$ & 0.789 \\
\hline Triglycerides (mg/dl) & $107.1 \pm 34.8$ & $119.5 \pm 27.9$ & 0.245 \\
\hline Uric acid (mg/dl) & $5.8 \pm 1.1$ & $5.4 \pm 0.8$ & 0.175 \\
\hline Serum creatinine (mg/dl) & $0.94 \pm 0.14$ & $0.99 \pm 0.20$ & 0.402 \\
\hline Glycaemia (mg/dl) & $95.7 \pm 11.2$ & $93.1 \pm 7.5$ & 0.407 \\
\hline MoCA test ES & $1.94 \pm 0.94$ & $1.89 \pm 1.08$ & 0.870 \\
\hline Word Match Testing ES & $2.00 \pm 0.97$ & $2.50 \pm 1.20$ & 0.178 \\
\hline Stroop test E ES & $2.22 \pm 0.88$ & $1.67 \pm 1.65$ & 0.215 \\
\hline Stroop test T ES & $2.67 \pm 0.91$ & $2.28 \pm 1.67$ & 0.392 \\
\hline EQ-VAS & $65.3 \pm 12.8$ & $68.1 \pm 12.8$ & 0.520 \\
\hline Augmentation Index (\%) & $14.19 \pm 10.00$ & $18.59 \pm 18.21$ & 0.400 \\
\hline \multicolumn{4}{|l|}{ Antihypertensive therapy } \\
\hline ACE-inhibitors & $2(11.1)$ & $2(11.1)$ & 1.000 \\
\hline ATII receptor blockers & $13(72.2)$ & $14(77.8)$ & 0.700 \\
\hline Beta-blockers & $4(22.2)$ & $5(27.8)$ & 0.700 \\
\hline Calcium channel antagonists & $7(38.9)$ & $6(33.6)$ & 0.729 \\
\hline Diuretics & $9(50.0)$ & $9(50.0)$ & 1.000 \\
\hline
\end{tabular}

Values are numbers (percentage) or means \pm standard deviation $\mathrm{SBP}=$ systolic blood pressure; $\mathrm{DBP}=$ diastolic blood pressure; $\mathrm{HDL}=$ high density lipoprotein; $L D L=$ low-density lipoprotein; $\mathrm{MoCa}=$ Montreal Cognitive Assessment; $\mathrm{ES}=$ equivalent score; $\mathrm{EQ}-\mathrm{VAS}=\mathrm{EQ}-5 \mathrm{D}$ test visual analogue scale

patients receiving the active nutraceutical compound (Table 2 and Fig. 1). When tested for the cognitive functions, the hypertensive patients in the active treatment group performed significantly better test than the placebo group, as compared to the baseline condition (Table 3 and Fig. 2). In particular, there was a significant improvement in the performance obtained at the MoCA test and Word Match Testing (Table 3 and Fig. 2) for patients receiving the nutraceutical active compound. With a trend toward significance, the same group of hypertensive patients executed the Stroop test with improved scores, whereas no variation was observed for patients receiving the placebo. The psychometric assessment with the EQ-VAS scale revealed a significant amelioration in the active treatment group (Table 3 and Fig. 3) but 
Table 2 Characteristics of patients at baseline and after 6 months of treatment

\begin{tabular}{|c|c|c|c|}
\hline & Baseline & 6-months & $\begin{array}{l}\text { Treatment } \\
\text { effect }^{\mathrm{a}}\end{array}$ \\
\hline \multicolumn{4}{|l|}{$\mathrm{SBP}(\mathrm{mmHg})$} \\
\hline Control group & $133.3 \pm 16.9$ & $133.1 \pm 15.7$ & 0.132 \\
\hline Active treatment group & $135.6 \pm 13.5$ & $130.6 \pm 11.4$ & \\
\hline \multicolumn{4}{|l|}{$\mathrm{DBP}(\mathrm{mmHg})$} \\
\hline Control group & $84.1 \pm 12.3$ & $83.1 \pm 8.8$ & 0.402 \\
\hline Active treatment group & $85.8 \pm 9.1$ & $81.5 \pm 8.5$ & \\
\hline \multicolumn{4}{|l|}{ Heart rate (bpm) } \\
\hline Control group & $68.8 \pm 6.8$ & $68.2 \pm 5.3$ & 0.209 \\
\hline Active treatment group & $66.4 \pm 7.9$ & $68.3 \pm 8.3$ & \\
\hline \multicolumn{4}{|l|}{$\begin{array}{l}\text { Total cholesterol } \\
(\mathrm{mg} / \mathrm{dl})\end{array}$} \\
\hline Control group & $198.2 \pm 23.7$ & $210.8 \pm 21.7$ & 0.132 \\
\hline Active treatment group & $201.9 \pm 27.5$ & $204.4 \pm 23.6$ & \\
\hline \multicolumn{4}{|l|}{ HDL cholesterol (mg/dl) } \\
\hline Control group & $47.6 \pm 7.9$ & $51.3 \pm 11.9$ & 0.740 \\
\hline Active treatment group & $51.3 \pm 7.2$ & $54.3 \pm 7.7$ & \\
\hline \multicolumn{4}{|l|}{ LDL cholesterol (mg/dl) } \\
\hline Control group & $129.2 \pm 26.1$ & $138.9 \pm 22.4$ & 0.179 \\
\hline Active treatment group & $126.7 \pm 30.4$ & $126.9 \pm 25.0$ & \\
\hline \multicolumn{4}{|l|}{ Triglycerides (mg/dl) } \\
\hline Control group & $107.1 \pm 34.8$ & $103.1 \pm 35.0$ & 0.946 \\
\hline Active treatment group & $119.5 \pm 27.9$ & $115.9 \pm 24.3$ & \\
\hline \multicolumn{4}{|l|}{ Glycaemia (mg/dl) } \\
\hline Control group & $95.7 \pm 11.2$ & $96.6 \pm 13.3$ & 0.746 \\
\hline Active treatment group & $93.1 \pm 7.5$ & $93.0 \pm 8.0$ & \\
\hline \multicolumn{4}{|l|}{$\begin{array}{l}\text { Augmentation } \\
\text { Index (\%) }\end{array}$} \\
\hline Control group & $14.19 \pm 10.00$ & $14.69 \pm 11.79$ & 0.028 \\
\hline Active treatment group & $18.59 \pm 18.21$ & $12.24 \pm 12.30$ & \\
\hline
\end{tabular}

Values are means \pm standard deviation

$\mathrm{SBP}=$ systolic blood pressure; $\mathrm{DBP}=$ diastolic blood pressure; $\mathrm{HDL}=$ high-density lipoprotein; LDL = low-density lipoprotein

${ }^{\mathrm{a}}=$ active treatment versus placebo, GLM analysis

not in the control group receiving placebo (Table 3 and Fig. 3).

When we analyzed the potential correlations between changes of clinical and biochemical parameters with changes in neuropsychological scores obtained in the active treatment group, a positive relationship between SBP variation (from baseline to 6 months values) and EQ-VAS change was observed ( $r=0.554, p=0.017$, Fig. 4).

\section{Discussion}

The present randomized, double blind, placebocontrolled study demonstrated that a 6-month treatment with the novel nutraceutical preparation $\left(\mathrm{AkP05}\right.$, IzzeK $\mathrm{K}^{\circ}$,

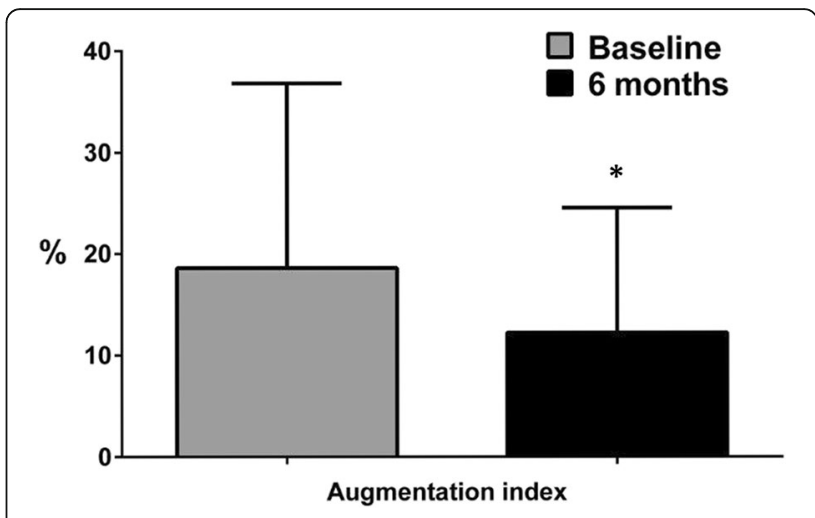

Fig. 1 Augmentation index at baseline and after 6 months in the active treated group. ${ }^{*} p=0.028$ for treatment effect (active treatment vs. placebo), GLM analysis

containing dry plant extracts of Bacopa monnieri, Ginkgo biloba and Green tea, plus Phosphatidylserine and Catechins, significantly improved the cognitive performance at MoCA test, Word Match Testing, Stroop test, and EQ-VAS, in a population of hypertensive patients on satisfactory blood pressure control. In contrast, the hypertensive patients treated with the placebo composition, had no significant modification in the scores obtained at the various cognitive tests. Thus, the improvement observed in patients treated with the active nutraceutical preparation could be ascribed to positive effects

Table 3 Characteristics of patients at baseline and after 6 months of treatment

\begin{tabular}{lccc}
\hline & Baseline & 6-months & Treatment effect $^{\mathrm{a}}$ \\
\hline MoCA test ES & & & \\
Control group & $1.94 \pm 0.94$ & $1.83 \pm 0.79$ & $<0.001$ \\
Active treatment group & $1.89 \pm 1.08$ & $2.89 \pm 1.08$ &
\end{tabular}

Word Match Testing ES

$\begin{array}{lll}\text { Control group } & 2.00 \pm 0.97 & 1.72 \pm 0.89 \\ \text { Active treatment group } & 2.50 \pm 1.20 & 2.94 \pm 1.21\end{array}$

Stroop test E ES

$$
\begin{array}{lll}
\text { Control group } & 2.22 \pm 0.88 & 1.94 \pm 1.11 \\
\text { Active treatment group } & 1.67 \pm 1.65 & 2.00 \pm 1.41
\end{array}
$$

Stroop test $\mathrm{T}$ ES

$$
\begin{array}{lll}
\text { Control group } & 2.67 \pm 0.91 & 2.22 \pm 1.11 \\
\text { Active treatment group } & 2.28 \pm 1.67 & 2.61 \pm 1.42
\end{array}
$$

EQ-VAS

$$
\begin{array}{lll}
\text { Control group } & 65.3 \pm 12.8 & 63.6 \pm 12.3 \\
\text { Active treatment group } & 68.1 \pm 12.8 & 80.6 \pm 12.0
\end{array}
$$

$<0.001$

Values are means \pm standard deviation

$\mathrm{MoCa}=$ Montreal Cognitive Assessment; $\mathrm{ES}=$ equivalent score; $\mathrm{EQ}-\mathrm{VAS}=\mathrm{EQ}-5 \mathrm{D}$ test visual analogue scale

${ }^{\mathrm{a}}=$ active treatment versus placebo, GLM analysis 


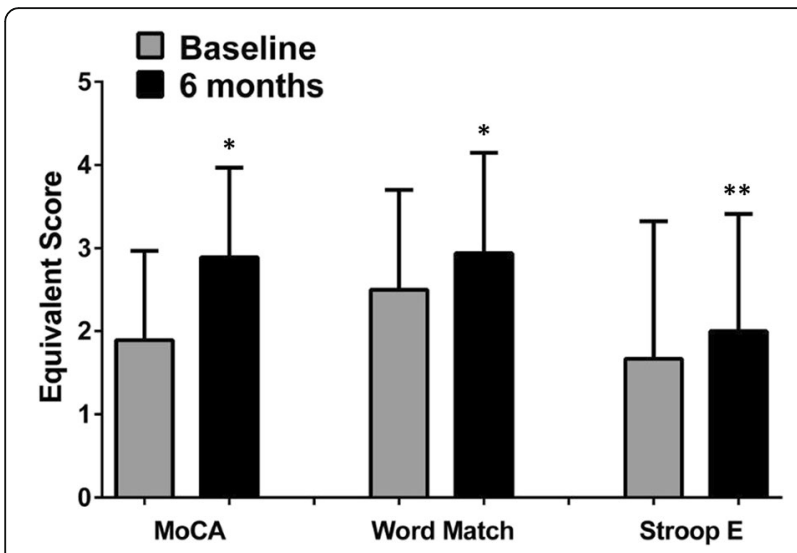

Fig. 2 Scores of neuropsychological tests at baseline and after 6 months in the active treated group. ${ }^{*} p<0.050$ for treatment effect (active treatment vs. placebo), GLM analysis. ** $p=0.050$ for treatment effect (active treatment vs. placebo), GLM analysis

on cognitive performance, ruling out the possibility of a placebo effect or potential habituation effects in performing the tests. In addition, hypertensive patients on active nutraceutical treatment displayed a significant improvement of arterial stiffness, as assessed by AI.

In this study, cognitive function was assessed accordingly to the most common measures used in general studies testing the impact of hypertension on cerebrovascular homeostasis. Basically, we tested how the nutraceutical compound under examination affected the most typically impaired cognitive domains of memory, executive function, and processing speed. The results obtained at the MoCA test after 6-months of treatment (active compound vs placebo), showed a significant improvement in the group of hypertensive patients receiving the nutraceutical preparation and no variation in the placebo group. Taking into consideration the fact that the battery of MoCA tests comprises a series of assessments capable to discriminate among different cognitive domains (memory and attention, visuospatial and executive functions, and

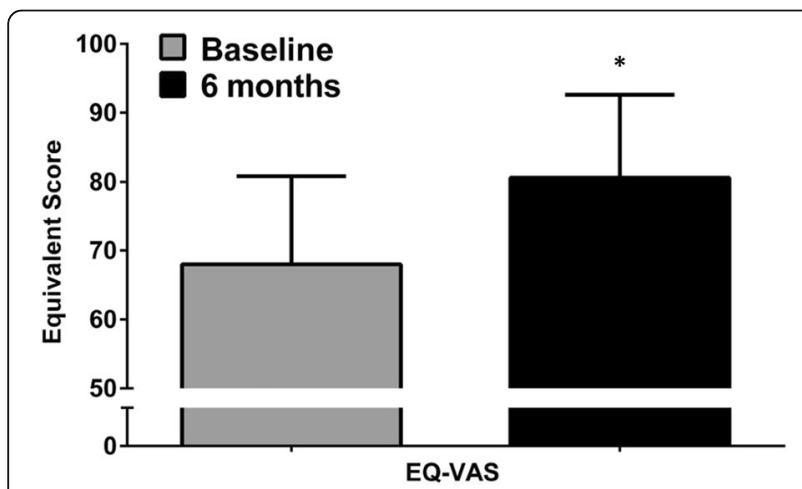

Fig. 3 Visual analogue scale (EQ-VAS) score at baseline and after 6 months in the active treated group. ${ }^{*} p<0.001$ for treatment effect (active treatment vs. placebo), GLM analysis

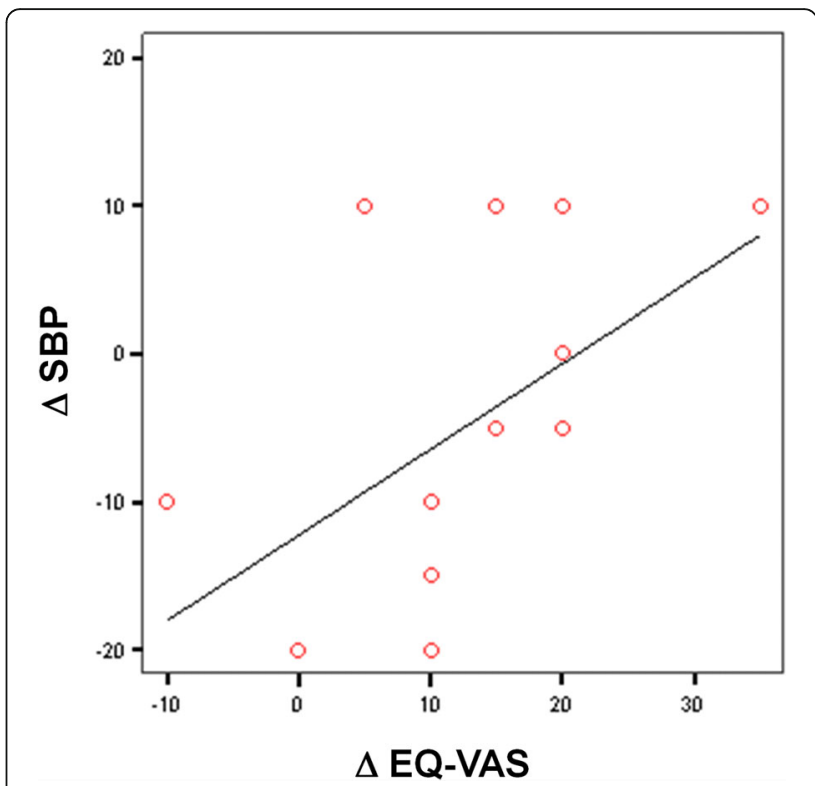

Fig. 4 Correlation between change in systolic blood pressure and change in visual analogue scale (EQ-VAS) after 6 months of treatment in the active treated group

language), we also evaluated more specific subdomains of cognition. Word-matching test highlighted a similar significant trend of improved learning abilities of the memory domain in patients receiving the active nutraceutical compound as compared to the placebo. The better performance in executive functions and processing speed was also confirmed when patients were subjected to the Stroop test, which showed enhanced functions in patients on active nutraceutical preparation. Lastly, the significantly increased score obtained in the VAS scale, indicative of patients' awareness on their health status, was also suggestive that the benefit obtained with the 6months treatment with the active nutraceutical compound was positively perceived by patients themselves.

Although several mechanisms may be involved in the improved overall cognitive function displayed by hypertensive patients treated with the nutraceutical compound, it could be envisaged that the concomitant reduction in arterial stiffness may be causally related. In fact, at the clinical level, indexes of aortic and large-artery stiffening are considered valuable predictors of cerebrovascular events and VCI [41, 42]. In this study, we observed that a 6months treatment with the active nutraceutical compound induced a significant reduction in the AI, a parameter correlated with arterial stiffness. No significant variation of the AI was found in the group of patients receiving the placebo for 6 months. Although we failed to detect significant correlations between the changes in $\mathrm{AI}$ and any of the measured cognitive parameters, we could speculate that the improvement in arterial stiffness positively influenced cerebral circulation, eventually enhancing cognitive functions. 
Chronic hypertension is also accompanied with hypertrophic remodeling of cerebral arteries and arterioles, a phenomenon well described in experimental models of hypertension and supported by evidence in patients. A critical aspect regarding cerebral circulation at the level of smaller arterioles consists in the disruption of the Blood-Brain Barrier (BBB), often leading to a potent inflammatory reaction, typically associated with the production of reactive oxygen species. A trait that can be commonly found in all the phenomena described above (hypertension, arterial stiffness, VCI and arterial stiffness) is the presence of a chronic low-grade inflammation, a process thought to contribute to pathogenesis of several diseases $[14,15]$. Several experimental evidence suggests that the use of nutraceutical compounds can be effective in hampering cognitive decline in experimental models [18-21]. Thus, the possibility that hypertension could influence onset/progression of late-life dementia/ sporadic AD by promoting neuroinflammatory processes through an increase in oxidative stress [43-46], accounts for the use of nutritional supplements with antioxidant action to counteract the development of cognitive decline in hypertensive patients. The nutraceutical preparation tested in the present study contains different molecules with potent antioxidant properties which, although with mixed results, have shown to have positive effects on cognitive function also in humans [22-32, 34], thus supporting the hypothesis that the improvement in cognitive function observed in our study is mediated by a reduction of the oxidative stress.

\section{Conclusions}

The potential to counteract cognitive decline in hypertensive patients is of utmost importance. Although some studies [47-50] have shown little benefit from using antioxidant or anti-hypertensive drugs, they are often generally not well accepted as long-term preventive tools by the general population. By contrast, the use of dietary supplements and nutraceutical preparations is becoming more common and widespread, since they are often seen as a useful tool, associated with a healthy lifestyle, to deal with health problems that inevitably arise over time, in the presence of predisposing risk factors.

In this study, we demonstrated that the therapy with a novel nutraceutical preparation (AkP05, IzzeK $\left.{ }^{\circ}\right)$ containing Bacopa monnieri, Ginkgo biloba, Phosphatidylserine, Green tea and Catechins is able to significantly increase the scores of important neuropsychological tests such as the MoCA battery of tests, the Word Match Testing and Stroop test. The positive variation in the EQ-VAS was also suggestive that patients increased their perception of a better health status. The fact that an enhancement of performance in specific cognitive domain was obtained in patients already on satisfactory control of blood pressure levels, could reflect that the effect was exerted at the level of target organ damage, probably supporting cerebrovascular function by reducing oxidative stress at the level of brain microcirculation. Future pharmacological and pathophysiological studies are needed to better clarify the mechanisms underlying this effect.

\section{Abbreviations \\ AD: Alzheimer's Disease; Al: augmentation index; $A \beta$ : amyloid $\beta$-peptide; BBB: Blood-Brain Barrier; BP: blood pressure ; DBP: diastolic blood pressure; ES: equivalent score; GLM: general linear model; MoCA: Montreal Cognitive Assessment; P1: first systolic peak; PP: pulse pressure; SBP: systolic blood pressure; VAS: Visual analogue scale; VCl: vascular cognitive impairment}

\section{Acknowledgements}

The authors wish to thank nurses Maria Abbate, Valeria Celestino, Maria Rosaria Cipro and Marco Colonna and their coordinator Anna Vitiello for their invaluable support in the management of patients.

\section{Funding}

The present research received no funding.

Availability of data and materials

Not applicable.

Authors' contributions

GG, AS, SDL, MLP, VDA, conceived and drafted the paper; AL, RI, VT made critical revisions to the draft. All authors read and approved the final manuscript.

Ethics approval and consent to participate

The study was approved by the Ethic Committee of the IRCCS Neuromed, Pozzilli (Isernia), Italy, which designed and registered the study protocol (ClinicalTrials.gov ID: NCT02572219).

Consent for publication

Not applicable.

\section{Competing interests}

The authors declare that they have no competing interests.

\section{Publisher's Note}

Springer Nature remains neutral with regard to jurisdictional claims in published maps and institutional affiliations.

\section{Author details}

${ }^{1}$ Hypertension Research Center; Department of Advanced Biomedical Sciences, Federico II University, Naples, Italy. ${ }^{2}$ Federico II University, Naples, Italy. ${ }^{3}$ Hypertension Research Center; Department of Translational Medical Sciences, Federico II University, via Pansini 5, 80131 Naples, Italy. ${ }^{4}$ Department of Angiocardioneurology and Translational Medicine, IRCCS Neuromed, Pozzilli, Isernia, Italy. ${ }^{5}$ Hypertension Research Center; Department of Neurosciences, Federico II University, Naples, Italy.

Received: 7 December 2017 Accepted: 28 December 2017

Published online: 07 February 2018

\section{References}

1. ladecola C, Yaffe K, Biller J, Bratzke LC, Faraci FM, Gorelick PB, Gulati M, Kamel H, Knopman DS, Launer $L$, et al. Impact of hypertension on cognitive function: a scientific statement from the American Heart Association. Hypertension. 2016;68(6):e67-94.

2. Prince M, Bryce R, Albanese E, Wimo A, Ribeiro W, Ferri CP. The global prevalence of dementia: a systematic review and metaanalysis. Alzheimers Dement. 2013;9(1):63-75. e62

3. Fotuhi $M$, Hachinski V, Whitehouse PJ. Changing perspectives regarding late-life dementia. Nat Rev Neurol. 2009;5(12):649-58.

4. Carnevale D, Mascio G, D'Andrea I, Fardella V, Bell RD, Branchi I, Pallante F, Zlokovic B, Yan SS, Lembo G. Hypertension induces brain beta-amyloid accumulation, cognitive impairment, and memory deterioration through 
activation of receptor for advanced glycation end products in brain vasculature. Hypertension. 2012;60(1):188-97.

5. Zlokovic BV. The blood-brain barrier in health and chronic neurodegenerative disorders. Neuron. 2008;57(2):178-201.

6. Deane R, Du Yan S, Submamaryan RK, LaRue B, Jovanovic S, Hogg E, Welch D, Manness L, Lin C, Yu J, et al. RAGE mediates amyloid-beta peptide transport across the blood-brain barrier and accumulation in brain. Nat Med. 2003;9(7):907-13.

7. Gorelick PB, Scuteri A, Black SE, Decarli C, Greenberg SM, ladecola C, Launer L, Laurent S, Lopez OL, Nyenhuis D, et al. Vascular contributions to cognitive impairment and dementia: a statement for healthcare professionals from the american heart association/american stroke association. Stroke. 2011;42(9):2672-713.

8. Carnevale D, Mascio G, Ajmone-Cat MA, D'Andrea I, Cifelli G, Madonna M, Cocozza G, Frati A, Carullo P, Carnevale L, et al. Role of neuroinflammation in hypertension-induced brain amyloid pathology. Neurobiol Aging. 2012; 33(1):205):e219-29.

9. Gentile MT, Poulet R, Di Pardo A, Cifelli G, Maffei A, Vecchione C, Passarelli F, Landolfi A, Carullo P, Lembo G. Beta-amyloid deposition in brain is enhanced in mouse models of arterial hypertension. Neurobiol Aging. 2009;30(2):222-8.

10. Faraco G, Park L, Zhou P, Luo W, Paul SM, Anrather J, ladecola C. Hypertension enhances Abeta-induced neurovascular dysfunction, promotes beta-secretase activity, and leads to amyloidogenic processing of APP. J Cereb Blood Flow Metab. 2016;36(1):241-52.

11. Spangenberg EE, Green KN. Inflammation in Alzheimer's disease: lessons learned from microglia-depletion models. Brain Behav Immun. 2017;61:1-11.

12. Faraco G, Sugiyama Y, Lane D, Garcia-Bonilla L, Chang H, Santisteban MM, Racchumi G, Murphy M, Van Rooijen N, Anrather J, et al. Perivascular macrophages mediate the neurovascular and cognitive dysfunction associated with hypertension. J Clin Invest. 2016;126(12):4674-89.

13. Park L, Uekawa K, Garcia-Bonilla L, Koizumi K, Murphy M, Pistik R, Younkin L, Younkin S, Zhou P, Carlson G, et al. Brain perivascular macrophages initiate the neurovascular dysfunction of Alzheimer Abeta peptides. Circ Res. 2017;121(3):258-69.

14. Minghetti $\mathrm{L}$. Role of inflammation in neurodegenerative diseases. Curr Opin Neurol. 2005;18(3):315-21.

15. Wyss-Coray T. Inflammation in Alzheimer disease: driving force, bystander or beneficial response? Nat Med. 2006;12(9):1005-15.

16. Li JJ, Chen JL. Inflammation may be a bridge connecting hypertension and atherosclerosis. Med Hypotheses. 2005;64(5):925-9.

17. Marvar PJ, Lob H, Vinh A, Zarreen F, Harrison DG. The central nervous system and inflammation in hypertension. Curr Opin Pharmacol. 2011;11(2):156-61.

18. Koh SH, Lee SM, Kim HY, Lee KY, Lee YJ, Kim HT, Kim J, Kim MH, Hwang MS,

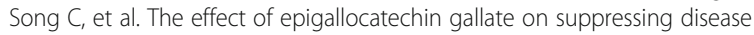
progression of ALS model mice. Neurosci Lett. 2006;395(2):103-7.

19. Rezai-Zadeh K, Arendash GW, Hou H, Fernandez F, Jensen M, Runfeldt M, Shytle RD, Tan J. Green tea epigallocatechin-3-gallate (EGCG) reduces beta-amyloid mediated cognitive impairment and modulates tau pathology in Alzheimer transgenic mice. Brain Res. 2008;1214:177-87.

20. Lee YK, Yuk DY, Lee JW, Lee SY, Ha TY, Oh KW, Yun YP, Hong JT. Epigallocatechin-3-gallate prevents lipopolysaccharide-induced elevation of beta-amyloid generation and memory deficiency. Brain Res. 2009;1250:164-74.

21. Araujo JA, Landsberg GM, Milgram NW, Miolo A. Improvement of shortterm memory performance in aged beagles by a nutraceutical supplement containing phosphatidylserine, Ginkgo Biloba, vitamin E, and pyridoxine. Can Vet J. 2008:49(4):379-85.

22. Stough C, Lloyd J, Clarke J, Downey LA, Hutchison CW, Rodgers T, Nathan PJ. The chronic effects of an extract of Bacopa monniera (Brahmi) on cognitive function in healthy human subjects. Psychopharmacology. 2001;156(4):481-4.

23. Roodenrys S, Booth D, Bulzomi S, Phipps A, Micallef C, Smoker J. Chronic effects of Brahmi (Bacopa Monnieri) on human memory. Neuropsychopharmacology. 2002;27(2):279-81.

24. Calabrese C, Gregory WL, Leo M, Kraemer D, Bone K, Oken B. Effects of a standardized Bacopa Monnieri extract on cognitive performance, anxiety, and depression in the elderly: a randomized, double-blind, placebocontrolled trial. J Altern Complement Med. 2008;14(6):707-13.

25. Morgan A, Stevens J. Does Bacopa Monnieri improve memory performance in older persons? Results of a randomized, placebo-controlled, double-blind trial. J Altern Complement Med. 2010;16(7):753-9.

26. Stough C, Clarke J, Lloyd J, Nathan PJ. Neuropsychological changes after 30-day Ginkgo Biloba administration in healthy participants. Int J Neuropsychopharmacol. 2001;4(2):131-4.
27. Schneider LS, DeKosky ST, Farlow MR, Tariot PN, Hoerr R, Kieser M. A randomized, double-blind, placebo-controlled trial of two doses of Ginkgo Biloba extract in dementia of the Alzheimer's type. Curr Alzheimer Res. 2005:2(5):541-51.

28. Woelk H, Arnoldt KH, Kieser M, Hoerr R. Ginkgo Biloba special extract EGb 761 in generalized anxiety disorder and adjustment disorder with anxious mood: a randomized, double-blind, placebo-controlled trial. J Psychiatr Res. 2007;41(6):472-80

29. Cenacchi T, Bertoldin T, Farina C, Fiori MG, Crepaldi G. Cognitive decline in the elderly: a double-blind, placebo-controlled multicenter study on efficacy of phosphatidylserine administration. Aging (Milano). 1993;5(2):123-33.

30. Kesse-Guyot E, Fezeu L, Andreeva VA, Touvier M, Scalbert A, Hercberg S, Galan P. Total and specific polyphenol intakes in midlife are associated with cognitive function measured 13 years later. J Nutr. 2012;142(1):76-83.

31. Mohamed S, Lee Ming T, Jaffri JM. Cognitive enhancement and neuroprotection by catechin-rich oil palm leaf extract supplement. J Sci Food Agric. 2013;93(4):819-27.

32. Snitz BE, O'Meara ES, Carlson MC, Arnold AM, Ives DG, Rapp SR, Saxton J, Lopez OL, Dunn LO, Sink KM, et al. Ginkgo Biloba for preventing cognitive decline in older adults: a randomized trial. JAMA. 2009; 302(24):2663-70.

33. Nurk E, Refsum H, Drevon CA, Tell GS, Nygaard HA, Engedal K, Smith AD. Intake of flavonoid-rich wine, tea, and chocolate by elderly men and women is associated with better cognitive test performance. J Nutr. 2009;139(1):120-7.

34. Kuriyama S, Hozawa A, Ohmori K, Shimazu T, Matsui T, Ebihara S, Awata S, Nagatomi R, Arai H, Tsuji I. Green tea consumption and cognitive function: a crosssectional study from the Tsurugaya project 1. Am J Clin Nutr. 2006;83(2):355-61.

35. Letenneur L, Proust-Lima C, Le Gouge A, Dartigues JF, Barberger-Gateau P. Flavonoid intake and cognitive decline over a 10-year period. Am J Epidemiol. 2007;165(12):1364-71.

36. Mastroiacovo D, Kwik-Uribe C, Grassi D, Necozione S, Raffaele A, Pistacchio L, Righetti R, Bocale R, Lechiara MC, Marini C, et al. Cocoa flavanol consumption improves cognitive function, blood pressure control, and metabolic profile in elderly subjects: the Cocoa, cognition, and aging (CoCoA) study-a randomized controlled trial. Am J Clin Nutr. 2015;101(3):538-48.

37. Mancia G, Fagard R, Narkiewicz K, Redon J, Zanchetti A, Bohm M, Christiaens T, Cifkova R, De Backer G, Dominiczak A, et al. 2013 ESH/ESC guidelines for the Management of Arterial Hypertension: the task force for the management of arterial hypertension of the European Society of Hypertension (ESH) and of the European Society of Cardiology (ESC). Eur Heart J. 2013;34(28):2159-219.

38. Popovic IM, Seric V, Demarin V. Mild cognitive impairment in symptomatic and asymptomatic cerebrovascular disease. J Neurol Sci. 2007;257(1-2):185-93.

39. Spinnler HTG. Standardizzazione e taratura italiana di test neuropsicologici. Ital J Neurol Sci. 1987;6:8-120.

40. Chen $\mathrm{CH}$, Nevo E, Fetics B, Pak PH, Yin FC, Maughan WL, Kass DA. Estimation of central aortic pressure waveform by mathematical transformation of radial tonometry pressure. Validation of generalized transfer function. Circulation. 1997;95(7):1827-36.

41. Matsumoto A, Satoh M, Kikuya M, Ohkubo T, Hirano M, Inoue R, Hashimoto T, Hara A, Hirose T, Obara T, et al. Day-to-day variability in home blood pressure is associated with cognitive decline: the Ohasama study. Hypertension. 2014;63(6):1333-8.

42. Bohm M, Schumacher $H$, Leong D, Mancia G, Unger T, Schmieder R, Custodis F, Diener HC, Laufs U, Lonn E, et al. Systolic blood pressure variation and mean heart rate is associated with cognitive dysfunction in patients with high cardiovascular risk. Hypertension. 2015;65(3):651-61.

43. Minuz P, Patrignani P, Gaino S, Degan M, Menapace L, Tommasoli R, Seta F, Capone ML, Tacconelli S, Palatresi S, et al. Increased oxidative stress and platelet activation in patients with hypertension and renovascular disease. Circulation. 2002;106(22):2800-5.

44. Morris MC, Evans DA, Tangney CC, Bienias JL, Wilson RS. Associations of vegetable and fruit consumption with age-related cognitive change. Neurology. 2006;67(8):1370-6.

45. Craft S, Foster TC, Landfield PW, Maier SF, Resnick SM, Yaffe K, Session III. Mechanisms of age-related cognitive change and targets for intervention: inflammatory, oxidative, and metabolic processes. J Gerontol A Biol Sci Med Sci. 2012;67(7):754-9.

46. Poulet R, Gentile MT, Vecchione C, Distaso M, Aretini A, Fratta L, Russo G, Echart C, Maffei A, De Simoni MG, et al. Acute hypertension induces oxidative stress in brain tissues. J Cereb Blood Flow Metab. 2006;26(2):253-62. 
47. Saxby BK, Harrington F, Wesnes KA, McKeith IG, Ford GA. Candesartan and cognitive decline in older patients with hypertension: a substudy of the SCOPE trial. Neurology. 2008;70(19 Pt 2):1858-66.

48. Hanon O, Berrou JP, Negre-Pages L, Goch JH, Nadhazi Z, Petrella R, Sedefdjian A, Sevenier F, Shlyakhto EV, Pathak A. Effects of hypertension therapy based on eprosartan on systolic arterial blood pressure and cognitive function: primary results of the observational study on cognitive function and systolic blood pressure reduction open-label study. J Hypertens. 2008;26(8):1642-50.

49. Anderson C, Teo K, Gao P, Arima H, Dans A, Unger T, Commerford P, Dyal L, Schumacher $\mathrm{H}$, Pogue J, et al. Renin-angiotensin system blockade and cognitive function in patients at high risk of cardiovascular disease: analysis of data from the ONTARGET and TRANSCEND studies. Lancet Neurol. 2011;10(1):43-53.

50. Katada E, Uematsu N, Takuma Y, Matsukawa N. Comparison of effects of valsartan and amlodipine on cognitive functions and auditory p300 eventrelated potentials in elderly hypertensive patients. Clin Neuropharmacol. 2014;37(5):129-32

Submit your next manuscript to BioMed Central and we will help you at every step:

- We accept pre-submission inquiries

- Our selector tool helps you to find the most relevant journal

- We provide round the clock customer support

- Convenient online submission

- Thorough peer review

- Inclusion in PubMed and all major indexing services

- Maximum visibility for your research

Submit your manuscript at www.biomedcentral.com/submit 